\title{
INTEGRAÇÃo ENTRE O DESENVOLVIMENTO DE NOVOS PRODUTOS E DA PRODUÇÃO PARA A ECONOMIA CIRCULAR: UMA ANÁLISE TEÓRICA
}

Ilton Marchi de Almeida (ilton.marchi@unesp.br) - Departamento de Engenharia de Produção / Faculdade de Engenharia de Bauru (FEB) / Universidade Estadual Paulista (UNESP).

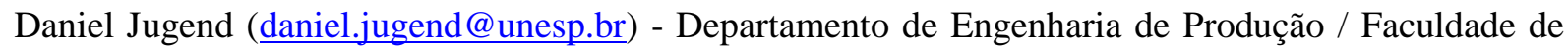
Engenharia de Bauru (FEB) / Universidade Estadual Paulista (UNESP).

Fernando Bernardi de Souza (fernando.bernardi@unesp.br) - Departamento de Engenharia de Produção / Faculdade de Engenharia de Bauru (FEB) / Universidade Estadual Paulista (UNESP).

\section{RESUMO}

Apesar da abordagem da economia circular (EC) ser uma das mais promissoras para operacionalizar o desenvolvimento sustentável, pouco se sabe sobre as suas relações com o desenvolvimento da produção. O desenvolvimento de produtos para a EC depende de um conjunto de premissas e expectativas associadas ao desenvolvimento da produção que ainda precisam ser mais investigadas. Este trabalho visa contribuir com o tema por meio de revisão sistemática e de conteúdo da literatura, o qual integra desenvolvimento do produto e da produção para a EC. Os resultados encontrados confirmam que a viabilidade dos princípios da EC, depende de modelos de negócios que ofereçam o compartilhamento de informações e de conhecimentos entre as organizações mediante a adoção de novas tecnologias como a Indústria 4.0 e blockchain.

Palavras chave: economia linear; economia circular; desenvolvimento de produto; desenvolvimento da produção. 


\section{INTRODUÇÃO}

As alterações climáticas das últimas décadas têm preocupado a sociedade com evidências visuais de degelo de áreas de geleiras permanentes, incêndios de grandes proporções, perda de biodiversidade e agressividade das intempéries em todas as partes do mundo (BALETA et al., 2019). As tendências dos eventos mostram que ações de recuperação ambiental precisam ser aplicadas para que o planeta não passe do limite de regeneração dos biomas globais. O apelo para ações com maior sustentabilidade para as ações humanas cresce na sociedade, alterando os comportamentos de consumo e os meios de produção. Composta por três dimensões que suportam as questões econômicas, sociais e ambientais; a sustentabilidade tem buscado o equilíbrio que garante o atendimento das necessidades do presente sem prejudicar as necessidades das gerações futuras (CHEN et al., 2020).

Órgãos intergovernamentais tem reagido as ameaças das consequências da degradação do meio ambiente e estabeleceram vários acordos rumo a maior sustentabilidade das ações humanas (CASTRO FILHO, 2018). A aderência à abordagem de recuperação ambiental tem recebido atenção da UE (União Europeia) que divulgou várias estratégias com o objetivo de conseguir maior sustentabilidade (HARTLEY; VAN SANTEN; KIRCHHERR, 2020). A UE defende que exista uma mudança de abordagem que migre da economia linear, focada na gestão unidirecional de materiais e energia, para sistemas circulares, denominada Economia Circular (ELLEN MACARTHUR FOUNDATION E MCKINSEY \& COMPANY, 2014). A gestão amplia a área de atuação da cadeia de suprimentos para ciclos fechados de gestão sistêmica dos fluxos de materiais e energia a fim de alcançar a prevenção de resíduos e aumentar a produtividade dos recursos (LOIZIA et al., 2021).

Expostos aos clientes, os produtos começaram a receber as influências dos movimentos ambientais e reagiram com iniciativas rumo a maior sustentabilidade (SUTHERLAND et al., 2020). Inicialmente, o eco-design surgiu como uma resposta às preocupações ambientais, mas alinhada com ganhos ambientais da economia linear (ARANDA-USÓN et al., 2020). Recentemente, os desenvolvimentos de produtos começaram a atender um maior número de requisitos que direcionam capacitações de circularidade de materiais e energia, aproximandose aos conceitos de funcionamento da EC (DEN HOLLANDER; BAKKER; HULTINK; 2017).

Elo obrigatório entre o desenvolvimento do produto e a estratégia das organizações, o desenvolvimento da produção deve perceber as alterações impostas pelos movimentos ambientais e seguir rumo às adaptações necessárias para o funcionamento em ambientes 
circulares (VOGT DUBERG et al., 2020). Na economia linear, o planejamento e controle da produção atua no contexto parcial de movimentação de materiais e energia, a chegada da circularidade impõe a ampliação da área de atuação e responsabilidade destas atividades nas organizações (JABBOUR et al., 2019; TSILIYANNIS, 2020).

Considerando os novos desafios impostos à área responsável pelo desenvolvimento e operação de sistemas de produção, este artigo avaliou relações entre desenvolvimento de novos produtos e desenvolvimento da produção sob a ótica da EC. A pesquisa analisou na base Scopus as publicações destas duas áreas, identificando similaridades e lacunas entre as iniciativas das duas abordagens de desenvolvimento. Após esta introdução, o artigo apresenta a revisão teórica (seção dois). A seção três descreve os critérios e protocolos de pesquisa utilizados. Posteriormente, resultados e discussões são apresentados. Finalmente, são delineadas as considerações finais s desse artigo (seção cinco).

\section{REVISÃO TEÓRICA}

\subsection{Economia circular}

A EC descreve um sistema econômico que troca o conceito de final de vida útil do produto por ações de redução, reuso, reciclagem e recuperação de materiais na produção, distribuição e consumo. Os princípios da EC podem se manifestar no nível micro (produtos, companhias, consumidores), intermediário (parques eco-industriais) e macro (cidades, regiões, nações) com o objetivo do desenvolvimento sustentável, que gera benefícios sistêmicos para o ambiente, economia e sociedade, atendendo demandas do presente sem comprometer oportunidades futuras (KIRCHHERR; REIKE; HEKKERT, 2017).

As Organizações das Nações Unidas (ONU) adotaram em 2015 um grupo de 17 metas globais interligadas de objetivos de desenvolvimento sustentável (ODS). Chamada de Agenda 2030, esta resolução da ONU trouxe na sua primeira versão objetivos amplos e interdependentes, que foram revisados em 2017 para o estabelecimento de metas específicas para cada objetivo e associadas a indicadores de acompanhamento.

Os princípios da EC associados ao design restaurador reúnem relacionamento direto com os indicadores de atendimento dos objetivos de desenvolvimento sustentável (Figura 1). Estudos indicam que as práticas de EC nos modelos de negócios podem ajudar a alcançar muitas metas de ODS, estando relacionadas diretamente a 21 e indiretamente a 28 indicadores dos 
objetivos de sustentabilidade (RODRIGUEZ-ANTON et al., 2019; SCHROEDER; ANGGRAENI; WEBER, 2019).

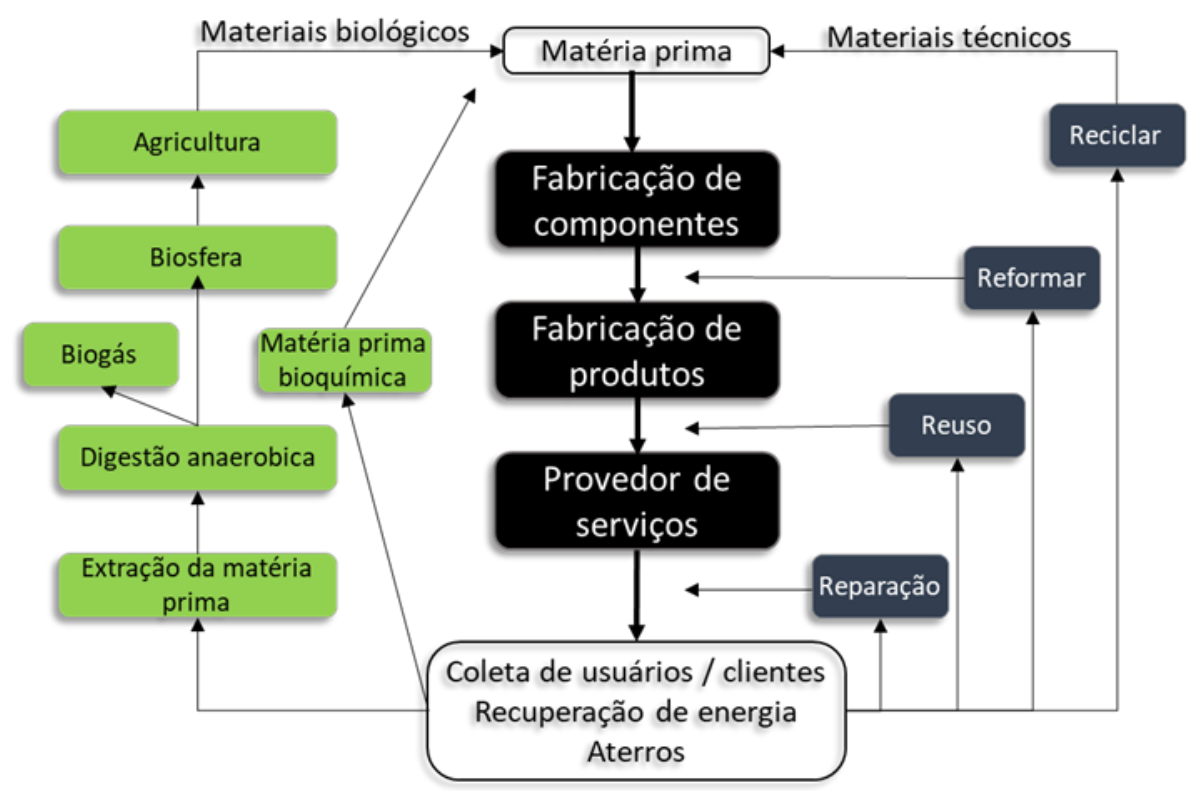

FIGURA 1 - Sistema industrial restaurador por design. Fonte: Ellen MacArthur Foundation e McKinsey \& Company (2014).

\subsection{Desenvolvimento de novos produtos}

As iniciativas do desenvolvimento de produto conforme os pressupostos do eco-design promoveram muitos avanços nos movimentos ambientais, priorizando o gerenciamento de resíduos com foco na prevenção e reaproveitamento (ARANDA-USÓN et al., 2020). Modificando o foco de atuação, o desenvolvimento de produto conforme as iniciativas de EC assume o pressuposto que o resíduo deixa de existir, imitando um sistema de ciclo fechado, onde os recursos que entram devem permanecer contabilizados em todos os momentos da vida útil do produto (Figura 2). O desenvolvimento do produto conforme os princípios da EC abrangem tanto a integridade do produto (destinado a prevenir e reverter a obsolescência a um nível de produto e componente) quanto o design para a reciclagem (destinado a prevenir e reverter a obsolescência) (DEN HOLLANDER; BAKKER; HULTINK, 2017). 


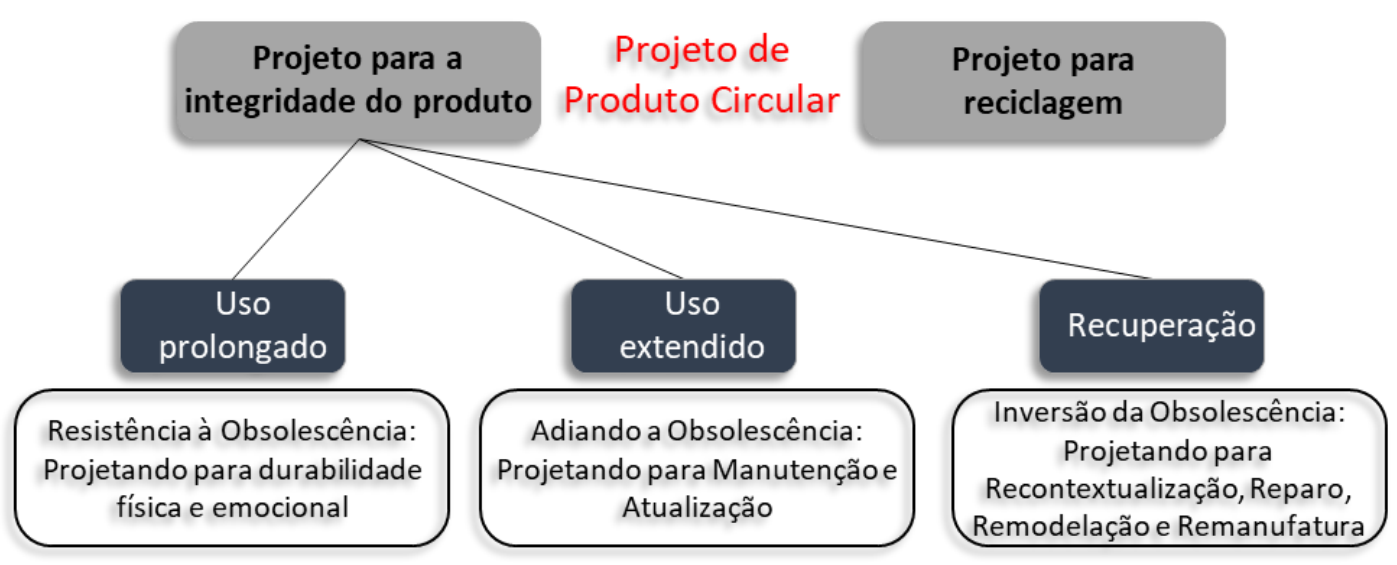

FIGURA 2 - Abordagens de projetos para produtos circulares. Fonte: Hollander, den, Bakker e Hultink (2017).

A revisão dos princípios envolvidos no desenvolvimento do produto transcende aos requisitos presentes no produto final e acrescentam movimentos sistêmicos de materiais e energia que circulam na cadeia expandida de suprimentos para a garantir o fechamento dos ciclos e a eliminação de geração de resíduos (JABBOUR et al., 2019).

\section{DESENVOLVIMENTO DA PRODUÇÃO}

As atividades de produção necessitam perceber as pressões e transformações em andamento nas áreas de produtos. Os novos requisitos de sustentabilidade exigidos aos produtos necessitam do suporte das atividades de produção (SUTHERLAND et al., 2020). Antes delimitadas às áreas internas da empresa, a produção expande os contornos de atuação acrescentando as operações da produção as novas capacidades de extensão de uso e recuperação de material e energia (VOGT DUBERG et al., 2020). A visão linear de ciclo aberto permitia um contexto menor de atuação com a descontinuidade da rastreabilidade além dos limites da cadeia de suprimentos. A aderências aos princípios de EC pressupõe a ausência de resíduos e todos os materiais e energia pertencem um ciclo fechado onde a cadeia de suprimento atua coordenadamente na circularidade de materiais biológicos e técnicos (HERCZEG; AKKERMAN; HAUSCHILD, 2018).

A adoção de iniciativas de EC apresenta comportamento progressivo que pode ser medido conforme as atividades correntes das empresas. A figura 3 apresenta 4 níveis de atividades relacionadas aos princípios de EC. Os estágios iniciais de adoção das práticas estão relacionados a recuperação de energia de materiais através de atividades como reciclagem. Os estágios mais avançados acompanham atividades coordenadas de simbiose industrial, nos 
quais os materiais seguem em loop fechado entre os participantes com redução efetiva de geração de resíduos (ARANDA-USÓN et al., 2020).

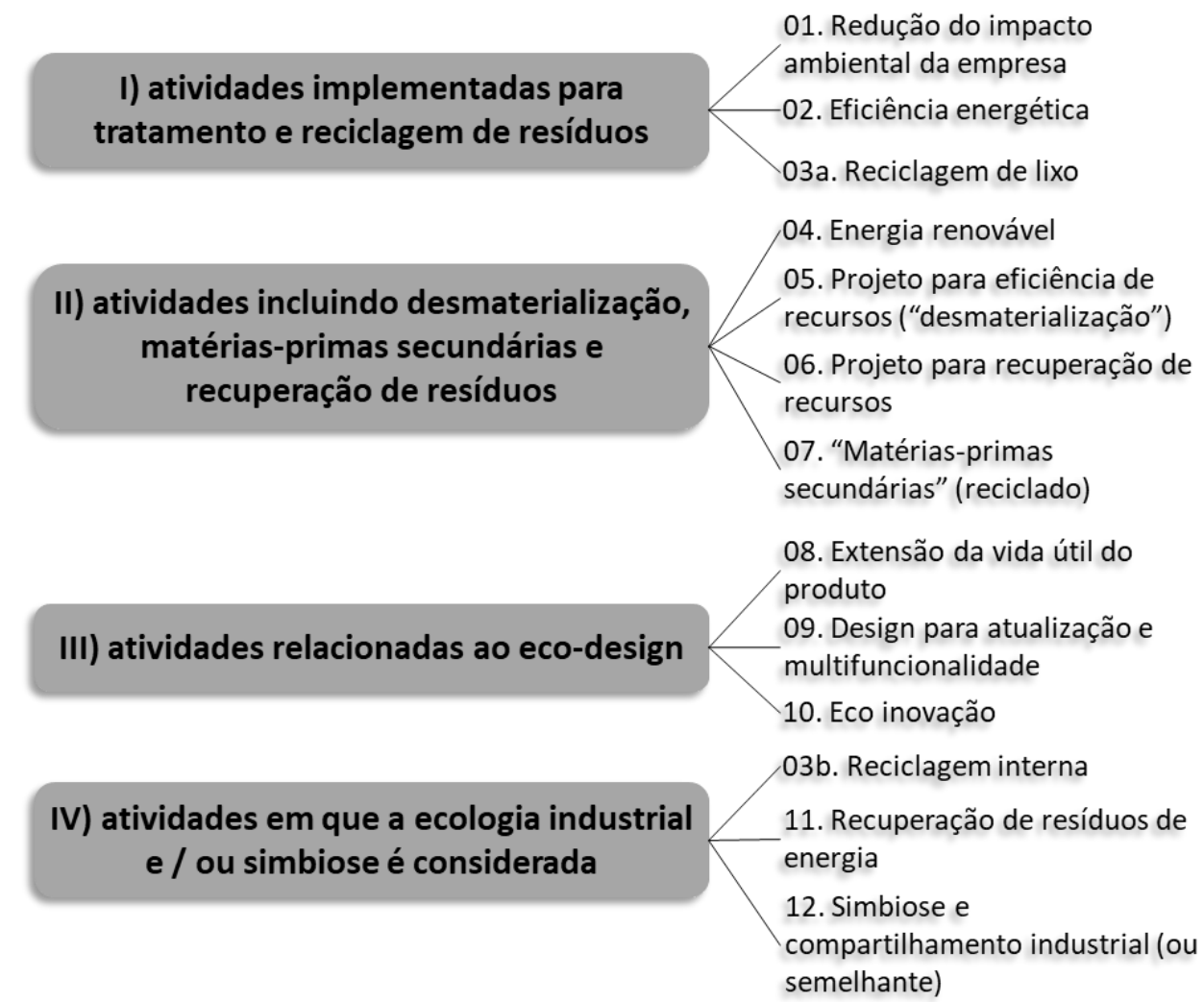

FIGURA 3 - Adoção progressiva da Economia Circular. Fonte: Aranda-Usón et al. (2020).

\section{METODO DE PESQUISA}

Considerando a relevância do montante e profundidade dos artigos disponíveis, esta pesquisa avaliou na base Scopus o comportamento de dois critérios de protocolo de pesquisa. As palavras chaves foram aplicadas para selecionar materiais relacionados aos dois grupos de artigos, assuntos associados ao desenvolvimento de produtos e desenvolvimento da produção, citados em artigos de abordagens de economia circular (Figura 4). 


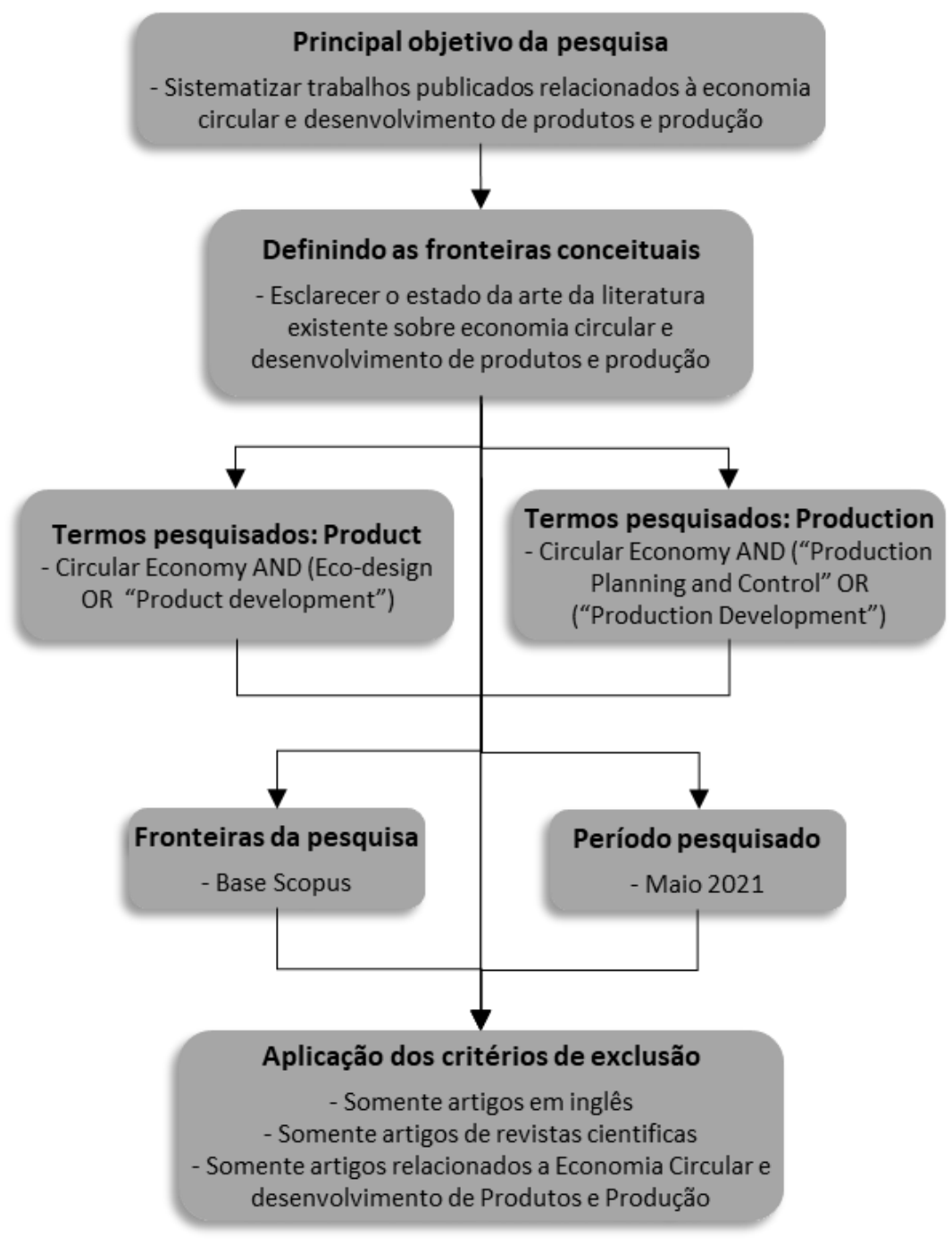

FIGURA 4 - Critério e protocolo da revisão de literatura.

\section{RESULTADOS E DISCUSSÕES}

O resultado da aplicação das palavras chaves nos dois grupos pesquisados apresentou grande desnível no número de registros. Enquanto o grupo de artigos de desenvolvimento de produtos apresentou um total de 101 artigos compatíveis com os critérios de pesquisa, somente 8 artigos foram identificados como compatíveis com o desenvolvimento da produção. A similaridade de comportamento dos dados se refere ao crescimento significativo do número de registros, sendo tendencia apresentada nos dois grupos estudados e acumulada nos últimos anos (Figura 5). 


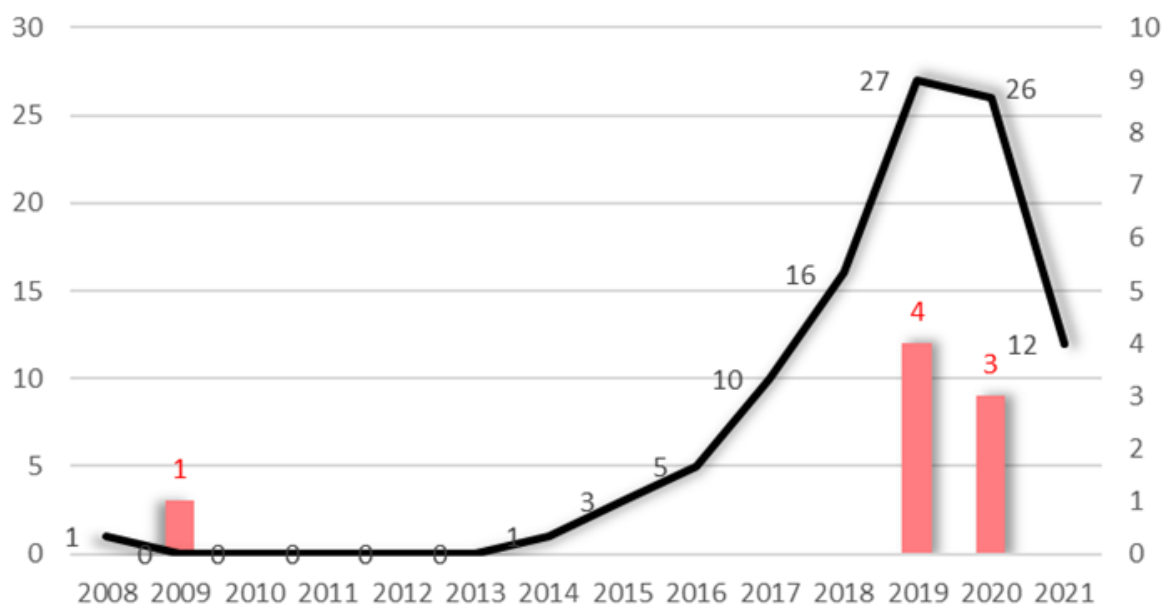

EC+Production $-E C+$ Product

FIGURA 5 - Concentração dos artigos conforme ano de publicação. Fonte: Scopus (2021).

Quando os dados são analisados quanto a região de geração dos artigos, não é percebida uma proporção na quantidade de artigos publicados. A concentração dos artigos compatíveis não obedeceu a uma proporção entre publicações de contexto associados a produtos equivalentes a criação de contexto associado a produção. Poucas manifestações regionais endereçaram proporcionalmente as preocupações de abordagens dos tipos de desenvolvimento (Figura 6).

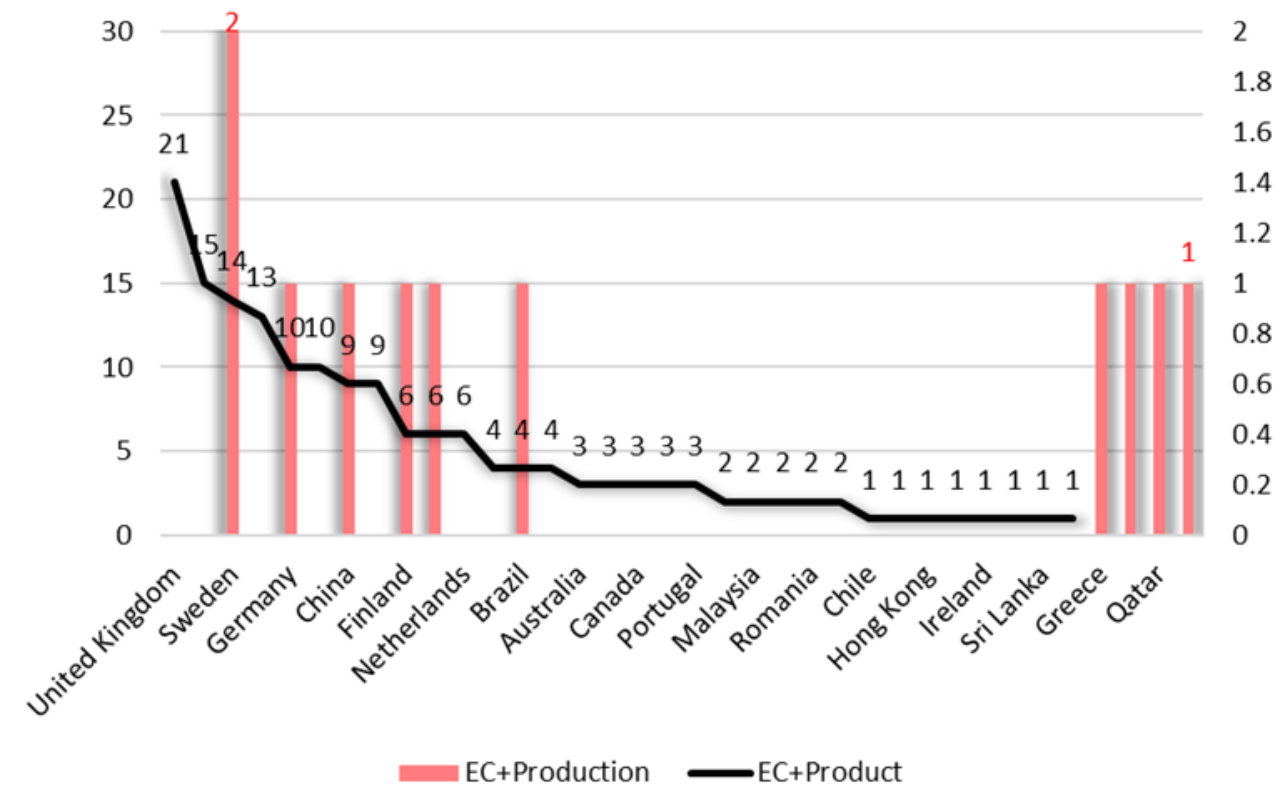

FIGURA 6 - Concentração dos artigos conforme país de publicação. Fonte: Scopus (2021). 
A visão das concentrações dos artigos conforme a área de pesquisa apresenta maior número de artigos de desenvolvimento de produtos associadas as áreas de pesquisas com maior proximidade de aspectos sociais. $\mathrm{O}$ comportamento das áreas que pesquisam $\mathrm{o}$ desenvolvimento da produção apresenta maior concentração em áreas associadas ao contexto econômico das pesquisas. Áreas de pesquisas como Engenharia e Ciência da computação do desenvolvimento da produção apresentaram as maiores relações de registros identificados, quando comparadas as publicações de desenvolvimento de produtos (Figura 7).

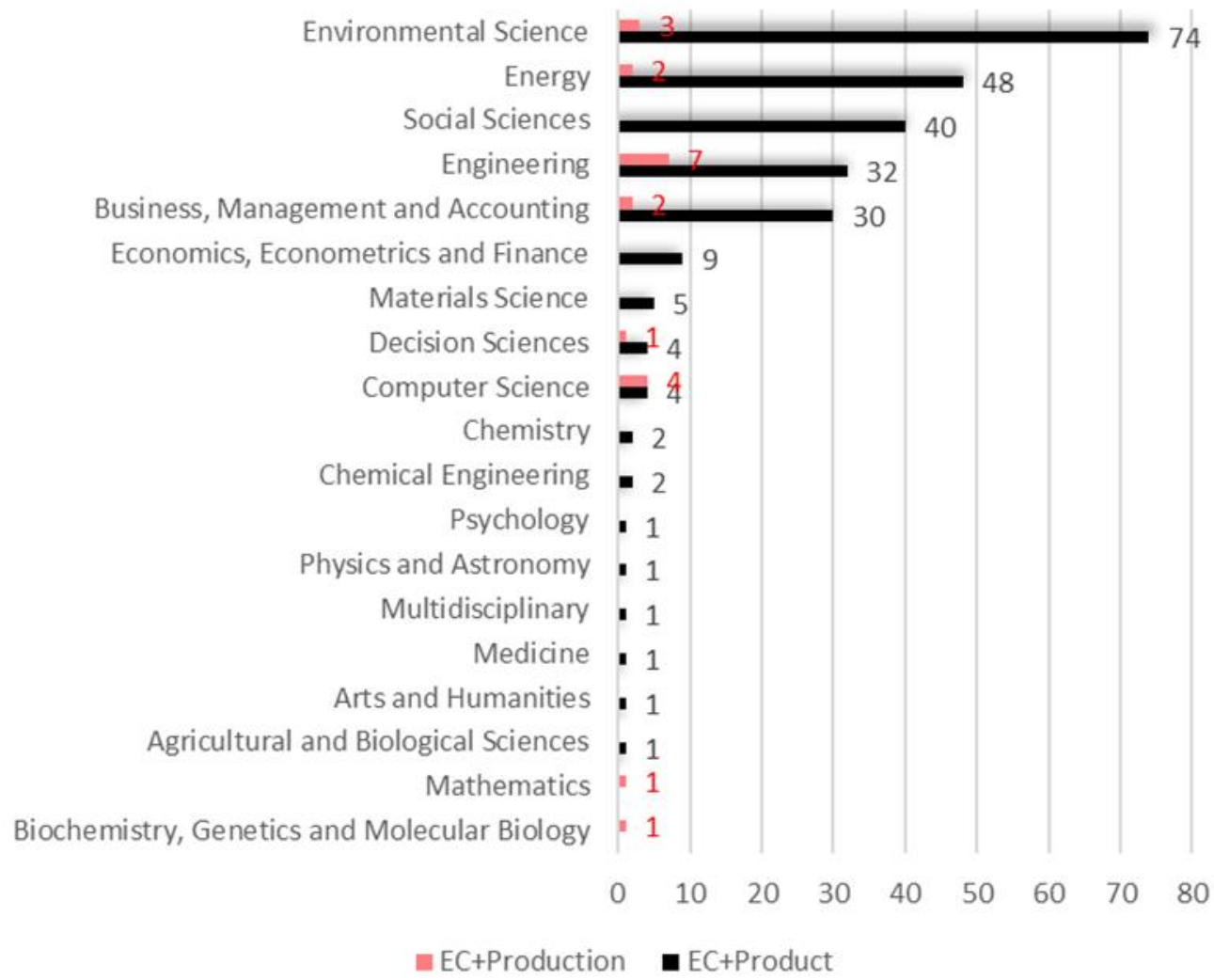

FIGURA 7 - Concentração dos artigos conforme área de pesquisa. Fonte: Scopus (2021).

A geração de ganhos de sustentabilidade significa avançar no sentido da criação equilibrada de benefícios nas dimensões econômicas, sociais e ambientais, atendendo as demandas do momento sem sacrificar oportunidades do futuro. Como consequência de níveis diferentes de exposições as pressões ambientais da sociedade, os setores envolvidos na cadeia de suprimentos de produtos manifestam reações desiquilibradas que limitam a capacidade sistêmica de geração de ganhos nas iniciativas para a geração de sustentabilidade (VOGT DUBERG et al., 2020). 
Os produtos são gerados e expostos aos seus clientes e recebem o impacto direto das manifestações das preocupações ambientais (SUTHERLAND et al., 2020). A maior quantidade de artigos publicados abordando o contexto de EC e desenvolvimento de produto pode suportar a interpretação de que a maior atenção foi dedicada ao bem final gerado, sem necessariamente tratar o contexto do desenvolvimento e operação do sistema de produção relacionada a geração do produto.

A análise bibliométrica dos dados da pesquisa identifica que existe a concentração de artigos gerados em países da CE (Comunidade Europeia) e, especialmente, o Reino Unido. Esta região assumiu posição de vanguarda nos movimentos ambientais e foi exposta às influências das metas de desenvolvimento sustentável demandadas pela sociedade e reguladas por agencias intergovernamentais (RODRIGUEZ-ANTON et al., 2019).

A discussão sobre um cenário no qual o produto precisa ser reformulado para conseguir maior sustentabilidade transcendem os limites do escopo do objeto criado (Figura 8). A agregação de especificidades que garantam maior longevidade, atributos para atualização e recuperação, exigem que exista um esforço sistêmico de capacitação do modelo de operação (LANZ et al., 2019). A estruturação da cadeia de suprimentos para atuar autocontida, sem permitir o vazamento de material e resíduo, deve evoluir proporcionalmente às transformações demandadas aos produtos (KALMYKOVA; SADAGOPAN; ROSADO, 2018).

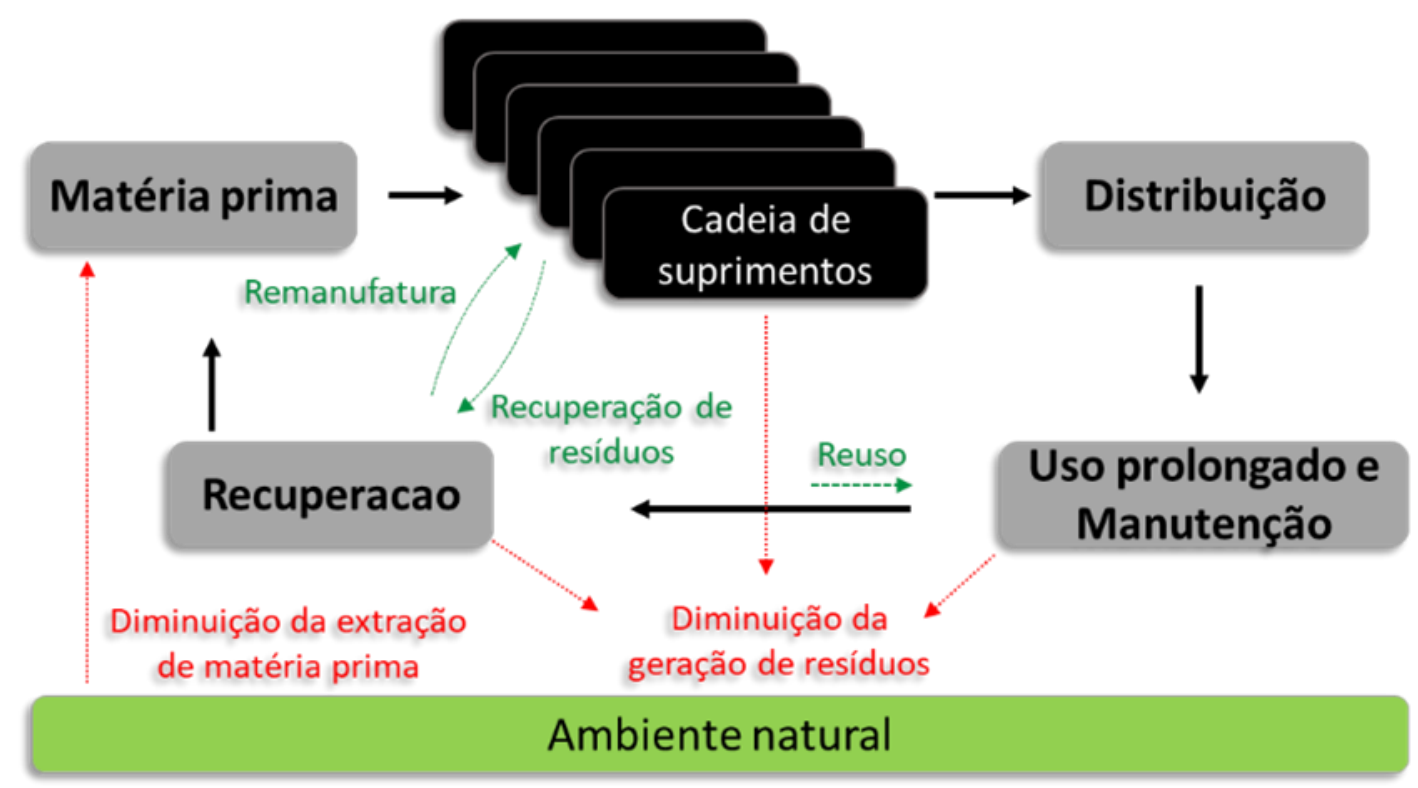

FIGURA 8 - Cadeia de suprimentos de ciclo fechado. Fonte: Fortună et al. (2012). 
Os processos e ferramentas que atendiam ao desenvolvimento tradicional da produção passam por questionamentos quanto à prontidão para atendimento dos cenários sustentáveis de atuação (SITCHARANGSIE; IJOMAH; WONG, 2019). As iniciativas de leitura de fluxo de material em tempo real e compartilhamento externo caminham em direção de tecnologias como a Indústria 4.0 e Blockchain (ESMAEILIAN et al., 2020; SUTHERLAND et al., 2020). A operação da cadeia de suprimentos de grandes organizações, mesmo as mais verticais e horizontais, transcendendo os limites de atuação direta. A viabilidade de atuações simbióticas demanda a externalidade de dados de fluxos de materiais entre os participantes (HERCZEG; AKKERMAN; HAUSCHILD, 2018).

A implementação da economia circular nas operações apresenta comportamento progressivo, atingindo níveis de proficiência à medida que a cadeia de suprimentos caminha no sentido de fechamento dos seus ciclos. Iniciativas de abertura de dados de circulação de material e energia, como os defendidos nos princípios da Inovação Aberta (BOGERS; CHESBROUGH; MOEDAS, 2018), podem acelerar a identificação de novas oportunidades de circulação e capacitação de novos participantes na cadeia de suprimentos, capazes de novos direcionamentos circulares aos produtos (TSENG; BUI, 2017). Contrariando a abordagem de fechamento da informação associada a identificação de novas descobertas, identificada como Inovação Fechada no Quadro 1, os princípios da "Inovação Aberta" combinam ideias internas e externas geradoras de valor que atendam um modelo de negócio (CHESBROUGH, 2003).

QUADRO 1 - Princípios de inovação fechada e aberta. 


\begin{tabular}{|c|c|}
\hline Princípios da inovação fechada & Princípios da inovação aberta \\
\hline $\begin{array}{l}\text { As pessoas inteligentes em nosso setor trabalham } \\
\text { para nós. }\end{array}$ & $\begin{array}{l}\text { Nem todas as pessoas inteligentes trabalham } \\
\text { para nós. }\end{array}$ \\
\hline $\begin{array}{l}\text { Para lucrar com P\&D*, nós devemos descobrir, } \\
\text { desenvolver e lançar uma nova inovação. }\end{array}$ & $\begin{array}{l}\text { P\&D* externo pode criar valor significativo; P\&D* } \\
\text { interno é necessário para resgatar parte do valor. }\end{array}$ \\
\hline $\begin{array}{l}\text { Se descobrirmos a inovação sozinhos, vamos } \\
\text { levar a descoberta ao mercado primeiro. }\end{array}$ & $\begin{array}{l}\text { Nós não temos que ser os descobridores da } \\
\text { inovação para lucrarmos com ela. }\end{array}$ \\
\hline $\begin{array}{l}\text { A organização vencerá se levar a inovação } \\
\text { primeiro ao mercado. }\end{array}$ & $\begin{array}{l}\text { A organização vencerá se fizer o melhor uso de } \\
\text { inovações internas e externas. }\end{array}$ \\
\hline $\begin{array}{l}\text { Devemos proteger e controlar a } \mathrm{PI}^{* *} \text {, assim o } \\
\text { concorrente não poderá lucrar com nossas ideias. }\end{array}$ & $\begin{array}{l}\text { Devemos lucrar com outros usos de nossas } \mathrm{PI}^{* *} \mathrm{e} \\
\text { deveríamos comprar outras } \mathrm{PI}^{* *} \text { se for um } \\
\text { avanço ao nosso modelo de negócio }\end{array}$ \\
\hline
\end{tabular}

\section{CONSIDERAÇÕES FINAIS}

Nos trabalhos em EC percebe-se um desnível de abordagem entre as pesquisas mais numerosas sobre o desenvolvimento de produtos e escassez de pesquisas que tratam as questões sobre desenvolvimento da produção. Uma visão parcial, focada somente no produto, sobre este contexto para as operações das organizações conforme os princípios de circularidade, é insuficiente para a aplicação das propostas encontradas na literatura em qualquer modelo de negócio. A utilização dos princípios de EC no desenvolvimento de produtos transcendem a ações de agregação de escopo aos produtos e demandam a contextualização de um novo modelo de operação, que modificam substancialmente a estratégia de atuação da produção. A viabilidade do modelo de operação com maior proficiência em sustentabilidade terá maiores avanços se as abordagens circulares tratarem o cenário completo dos desenvolvimentos de produto e produção.

O progresso de implementação dos princípios de circularidade nas cadeias de suprimentos depende da coordenação simbiótica de atores na busca da rastreabilidade do fluxo completo de materiais e energia. Os participantes da cadeia de suprimentos serão demandados para oferecer maior transparência nas suas operações, para aumentar as chances para que outras organizações descubram novas destinações para os materiais e energia que até aquele momento seriam descartados como resíduos.

O compartilhamento dos dados das empresas abre novas perspectivas de identificação de oportunidades fora do atual mercado da organização. A implicação desta nova demanda circular é que a capacidade de desenvolvimento atual deve ser aprimorada e amplificada conforme os atuais modelos de negócios, buscando novas aplicações para as descobertas que 
não se encaixam nas operações atuais, estejam elas internas ou externas à sua atual área de atuação.

O cenário descrito nesta pesquisa retrata um tema relativamente recente, que ainda apresenta a necessidade de esclarecimentos quanto as suas definições e princípios. Destaca-se, nesse sentido, que essa pesquisa possui como principal limitação o fato de ser totalmente teórica e que utilizou apenas a base de dados Scopus. Pesquisas futuras poderiam, portanto, ampliar a base de dados consultadas, assim como incluir outras palavras chaves de busca.

Investigações qualitativas futuras, por meio de estudo de casos, também poderiam ser úteis ao identificar novas relações entre o desenvolvimento de produtos para a e EC com os processos de desenvolvimento da produção, tais como os de manufatura e planejamento e controle da produção. A partir do progresso dos entendimentos sobre os desenvolvimentos conforme a EC, as próximas pesquisas deverão investir em alternativas para ampliações nas oportunidades de ciclos fechados. Neste contexto, as novas tecnologias de rastreabilidade, compartilhamento de dados e conhecimento seguirão como fontes promissoras de geração de maior sustentabilidade.

\section{REFERÊNCIAS}

ARANDA-USÓN, A.; PORTILLO-TARRAGONA, P.; SCARPELLINI, S. et al. The progressive adoption of a circular economy by businesses for cleaner production: An approach from a regional study in Spain. Journal of Cleaner Production, v. 247, 2020.

BALETA, J.; MIKULČIĆ, H.; KLEMEŠ, J. J. et al. Integration of energy, water and environmental systems for a sustainable development. Journal of Cleaner Production, 2019.

BOGERS, M.; CHESBROUGH, H.; MOEDAS, C. Open innovation: Research, practices, and policies. California Management Review, v. 60, n. 2, 2018.

CASTRO FILHO, C. M. The 2030 agenda for sustainable development: A reading of public policy in the key of the school library. Revista Digital de Biblioteconomia e Ciência da Informação, v. 16, n. 3, 2018.

CHEN, T. L.; KIM, H.; PAN, S.Y. et al. Implementation of green chemistry principles in circular economy system towards sustainable development goals: Challenges and perspectives. Science of the Total Environment, v. 716, 2020.

CHESBROUGH, H. The logic of open innovation: Managing intellectual property. California Management Review, 2003.

ELLEN MACARTHUR FOUNDATION; MCKINSEY \& COMPANY. Towards the Circular Economy: Accelerating the scale-up across global supply chains. World Economic Forum, 2014.

ESMAEILIAN, B.; SARKIS, J.; LEWIS, K. et al. Blockchain for the future of sustainable supply chain management in Industry 4.0. Resources, Conservation and Recycling, v. 163, 2020.

DEN HOLlANDER, M. C.; BAKKER, C. A.; HULTINK, E. J. Product Design in a Circular Economy: Development of a Typology of Key Concepts and Terms. Journal of Industrial Ecology, v. 21, n. 3, 2017.

FORTUNǍ, M. E.; SIMION, I. M.; GHINEA, C. et al. Analysis and management of specific processes from environmental engineering and protection based on sustainability indicators. Environmental Engineering and Management Journal, v. 11, n. 2, 2012. 
HARTLEY, K.; VAN SANTEN, R.; KIRCHHERR, J. Policies for transitioning towards a circular economy: Expectations from the European Union (EU). Resources, Conservation and Recycling, v. 155, 2020.

HERCZEG, G.; AKKERMAN, R.; HAUSCHILD, M. Z. Supply chain collaboration in industrial symbiosis networks. Journal of Cleaner Production, v. 171, 2018.

JABBOUR, A. B. L. S.; ROJAS LUIZ, J. V.; ROJAS LUIZ, O. et al. Circular economy business models and operations management. Journal of Cleaner Production, v. 235, p. 1525-1539, 2019.

KALMYKOVA, Y.; SADAGOPAN, M.; ROSADO, L. Circular economy - From review of theories and practices to development of implementation tools. Resources, Conservation and Recycling, v. 135, 2018.

KIRCHHERR, J.; REIKE, D.; HEKKERT, M. Conceptualizing the circular economy: An analysis of 114 definitions. Resources, Conservation and Recycling, 2017.

LANZ, M.; NYLUND, H.; LEHTONEN, T. et al. Circular economy in integrated product and production development education. Procedia Manufacturing. Anais...2019

LOIZIA, P.; VOUKKALI, I.; ZORPAS, A. A. et al. Measuring the level of environmental performance in insular areas, through key performed indicators, in the framework of waste strategy development. Science of the Total Environment, v. 753, 2021.

RODRIGUEZ-ANTON, J. M.; RUBIO-ANDRADA, L.; CELEMÍN-PEDROCHE, M. S. et al. Analysis of the relations between circular economy and sustainable development goals. International Journal of Sustainable Development and World Ecology, v. 26, n. 8, 2019.

SCHROEDER, P.; ANGGRAENI, K.; WEBER, U. The Relevance of Circular Economy Practices to the Sustainable Development Goals. Journal of Industrial Ecology, v. 23, n. 1, 2019.

SITCHARANGSIE, S.; IJOMAH, W.; WONG, T. C. Decision makings in key remanufacturing activities to optimise remanufacturing outcomes: A review. Journal of Cleaner Production, 2019.

SUTHERLAND, J. W.; SKERLOS, S. J.; HAAPALA, K. R. et al. Industrial Sustainability: Reviewing the Past and Envisioning the Future. Journal of Manufacturing Science and Engineering, v. 142, n. 11, 2020.

TSENG, M. L.; BUI, T. D. Identifying eco-innovation in industrial symbiosis under linguistic preferences: A novel hierarchical approach. Journal of Cleaner Production, v. 140, 2017.

TSILIYANNIS, C. A. Prognosis of product take-back for enhanced remanufacturing. Journal of Remanufacturing, v. 10, n. 1, p. 15-42, 2020.

VOGT DUBERG, J.; JOHANSSON, G.; SUNDIN, E. et al. Prerequisite factors for original equipment manufacturer remanufacturing. Journal of Cleaner Production, v. 270, 2020. 